\title{
Sustainable Human Presence on the Moon Using In Situ Resources
}

\author{
Carole A. McLemore ${ }^{1}$, John C. Fikes ${ }^{2}$, Kevin S. McCarley ${ }^{3}$, Charles A. Darby ${ }^{4}$, and Peter A. Curreri ${ }^{5}$ \\ National Aeronautics and Space Administration/Marshall Space Flight Center (MSFC), \\ Huntsville, Alabama, 35812 \\ James P. Kennedy ${ }^{6}$ and James E. Good ${ }^{7}$ \\ Teledyne Brown Engineering, Huntsville, Alabama, 35805 \\ and \\ Scott D. Gilley ${ }^{8}$ \\ Tec-Masters, Inc., Huntsville, Alabama, 35806
}

\begin{abstract}
New capabilities, technologies and infrastructure must be developed to enable a sustained human presence on the moon and beyond. The key to having this permanent presence is the utilization of in situ resources. To this end, NASA is investigating how in situ resources can be utilized to improve mission success by reducing up-mass, improving safety, reducing risk, and bringing down costs for the overall mission. To ensure that this capability is available when needed, technology development is required now. NASA/Marshall Space Flight Center (MSFC) is supporting this endeavor, along with other NASA centers, by exploring how lunar regolith can be mined for uses such as construction, life support, propulsion, power, and fabrication. Efforts at MSFC include development of lunar regolith simulant for hardware testing and development, extraction of oxygen and other materials from the lunar regolith, production of parts and tools on the moon from local materials or from provisioned feedstocks, and capabilities to show that produced parts are "ready for use". This paper discusses the lunar regolith, how the regolith is being replicated in the development of simulants and possible uses of the regolith.
\end{abstract}

\section{Introduction}

$\mathrm{T}$ HE U.S. Space Exploration Policy lays out a plan that includes manned missions to return to the moon, and to use the lunar missions as testbeds and stepping stones to manned missions beyond. To accomplish this, one or more outposts will have to be established on the moon, with long-term habitation, and the moon will be used to provide planning, training, and technology development for the missions elsewhere in our solar system ${ }^{1}$. Because the future lunar astronauts will be going not to plant "flags and footprints" but to colonize, it will be necessary to make maximum use of the resources found locally. Figure 1 provides a possible roadmap to go from the lunar regolith to oxygen and finally metal parts.

\footnotetext{
${ }^{1}$ Project Manager, ISRU/ISFR/Dust, Exploration Advanced Capabilities Office, VP33.

${ }^{2}$ Deputy Project Manager, ISRU/ISFR/Dust, Exploration Advanced Capabilities Office, VP33.

${ }^{3}$ Former Lead Systems Engineer, Systems Engineering Branch, ES13.

${ }^{4}$ Lead Systems Engineer, Project Engineering Branch, ES11, AIAA Senior Member.

${ }^{5}$ Project Scientist, Metals Engineering Branch, EM30.

${ }^{6}$ Repair \& Nondestructive Evaluations Technologies Team Lead, Aerospace Systems, M/S 60.

${ }^{7}$ Fabrication Technologies Engineer, Aerospace Systems, M/S 60.

${ }^{8}$ Fabrication Technologies Team Lead, Advanced Technology and Space Programs Directorate, Teledyne Brown Engineering M/S 60 .
} 
In addition, this will allow us to test methods of making use of the resources found on other bodies in our solar system, which will be even more necessary as we move mankind's frontiers beyond Earth's planetary system.

The National Aeronautics and Space Administration (NASA) is investigating how these "local" (in situ) resources can be utilized to extend mission capabilities, to reduce risk and to bring down overall costs. This can be done because of the enormous "mass leverage" obtained by using resources found on the moon, rather

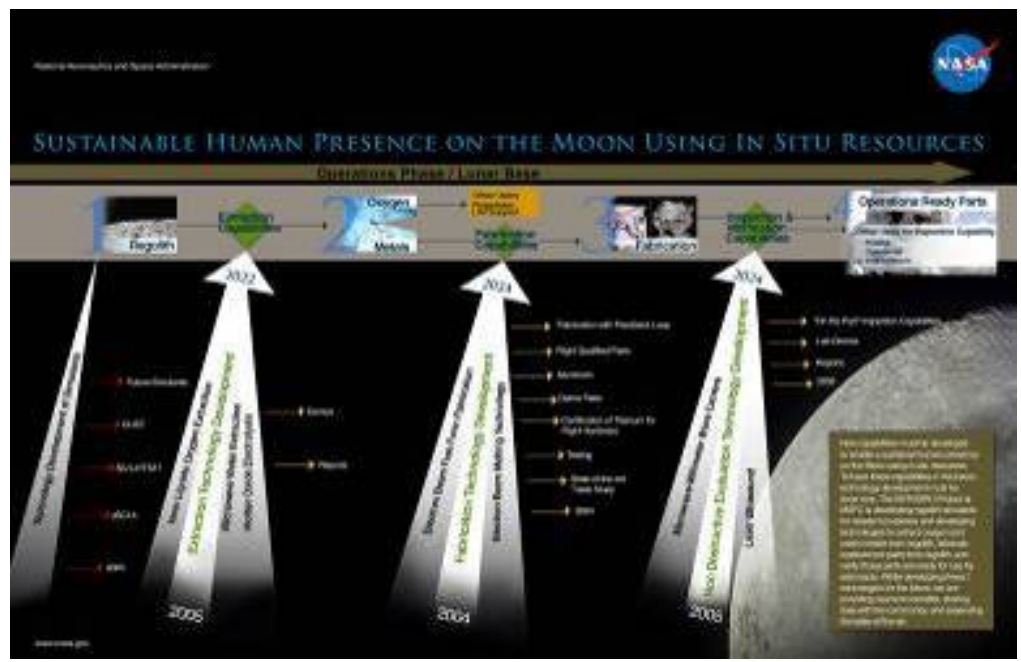

Figure 1. Lunar Resource Utilization Roadmap than carrying all needed materials from Earth. Safety margins are improved because oxygen, parts and tools can be produced locally as contingencies arise. Reduction in the mass that must be carried from Earth allows mass margins to be utilized for other equipment that cannot be produced locally, and the need to transport less mass translates into fewer missions needed to establish the lunar outposts, and, thus, amounts to cost savings. Figure 2 depicts a conceptual rendering of an ISRU processing facility.

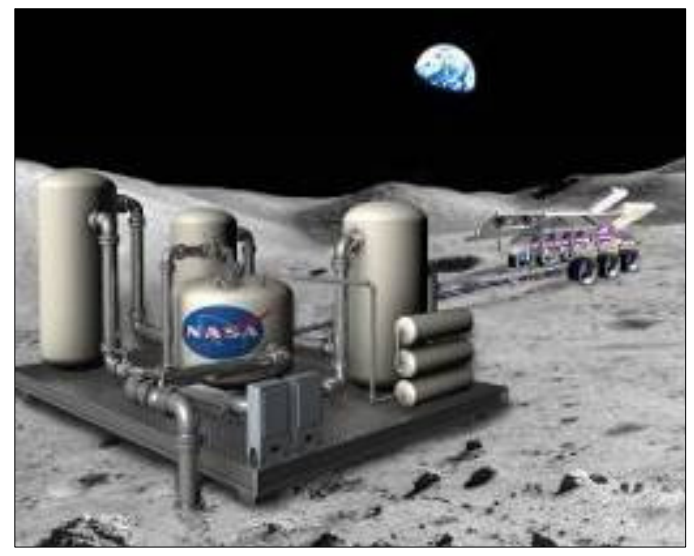

Figure 2. ISRU Processing Facility
NASA established a coordinated effort for ISRU ${ }^{4}$, managed at Johnson Space Center (JSC) with the participation of multiple Centers. The George C. Marshall Space Flight Center (MSFC) is one of the Centers participating in this effort and is exploring how to produce oxygen on the moon, how to capture and utilize metals and other products obtained from the spent regolith, and how to create parts from that metal resource.

The MSFC ISRU/ISFR/Dust Project is directing and coordinating the efforts to produce lunar dust and regolith simulants of the lunar regolith material, is performing research on the extraction of oxygen and other resources, is working to develop and certify a process for production of metallic tools and parts on the lunar surface, and is researching methods to examine and analyze those manufactured pieces through nondestructive evaluation so that they can be accepted for use. Each of these efforts is a vital piece in the integrated ISRU process, and is being coordinated with efforts underway at other NASA Centers and elsewhere to produce a complete end-to-end ISRU capability.

\section{Lunar Regolith and Simulants}

Less than 383 kilograms of lunar soil, rocks and core samples were returned from the six manned Apollo landing missions and three automated Soviet return craft. The picture of an Apollo astronaut obtaining a core sample during the Apollo 16 mission is shown in Figure 3. 
These samples have been extensively studied over the years ${ }^{2}$. As the technologies required to glean new data and information have evolved, much is still unknown. In addition, these samples represent only nine locations on the lunar surface, and, thus, cannot be assumed to represent the full range of lunar soil properties. However, much is known from these samples. Some samples of the soil contain relatively high concentrations of minerals such as ilmenite, are rich in metals like titanium and iron, and contain a high mass fraction of oxygen ${ }^{3}$. All of these components are valuable, local (in situ) resources that should be utilized to the maximum extent possible for the permanent lunar outpost(s).

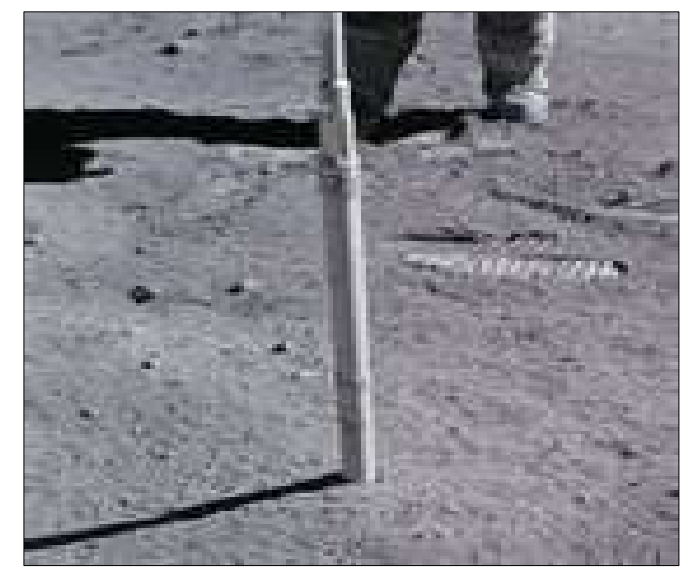

Figure 3. Apollo 16 Sample Drill Core

Lunar dust will be especially difficult to deal with, as evidenced by the experiences of the Apollo missions. Mitigation against the potential harmful effects of dust is of real concern. It is of paramount importance to understand the lunar regolith and its properties in order to develop robust and reliable systems and infrastructures with crew safety as the number one priority. Toxicology studies and development and verification tests must be performed on Earth prior to launching to the moon for extended lunar stays and eventually for missions to Mars.

Because there are only limited quantities of actual lunar samples that were brought down to Earth from the Apollo missions, simulants must be produced from terrestrial materials now to understand how to deal with the challenges of working with the lunar material upon return,. These simulants must mimic or represent as closely as possible the properties of actual lunar material, taking into account the known and unknown properties and variations. In the 1980s and 1990s, two such simulants were produced, MLS-1 (by the University of Minnesota) and JSC-1 (by the Johnson Space Center). Both of these were designed to simulate the lunar mare regions. Since that time,JSC-1 became a de facto standard for development of processes for lunar applications, but quantities were limited, and beginning in 2004 with the new emphasis on lunar exploration, , supplies were quickly exhausted.

Working under the NASA Human Systems Research and Technology Division, the MSFC ISRU team was given the lead for production of a new range of lunar simulants. The first to be produced was to be a "replacement" for JSC-1, having properties as close as reasonably possible to that simulant. This was the simulant previously used for the development work considered the baseline at that time. This new simulant of the lunar mare regions, named JSC-1A, started production in 2005 under a contract with Orbital Technologies, Incorporated (See Figure 4).

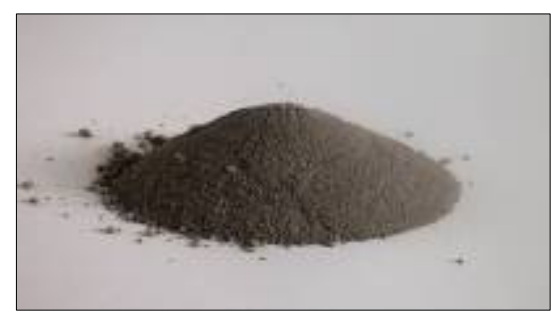

Figure 4. JSC-1A Simulant
Other simulants followed including some produced under the leadership of MSFC; some developed by private companies; and some by international partners. The most recent simulants developed under the leadership of MSFC include a series of simulants produced in partnership with the U.S. Geological Survey (USGS). These particular simulants, known as the NU-LHT series for NASA/U.S. Geological Survey Lunar Highlands Type, are designed to simulate properties of the lunar highlands region. A pilot dust simulant has also been produced in small quantities for the Dust Mitigation Project (see Figure 5). 
These new simulants are being developed under the NASA Exploration Technology Development Program (ETDP) in limited quantities. Based on the simulant users' needs, including the needs of the Constellation project, ETDP projects, and Human Health projects, a plan is being developed for the production of larger quantities of simulant based

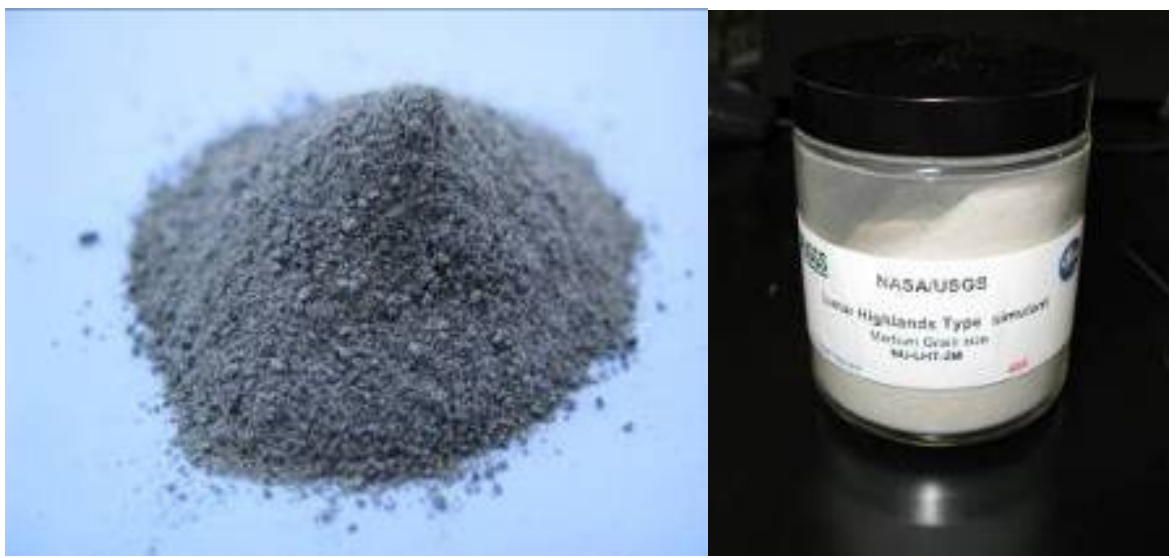

Figure 5. NU-LHT-1M and Jar of NU-LHT-2M Simulants on phased demands.

Because users will have different simulant requirements, MSFC continues to work with the user community to determine the level of simulant fidelity and the required simulant properties (chemical, physical, and other) to meet their specific application needs. For example, testing methods of oxygen extraction require exacting chemical properties, while tests of the behavior of vehicles in lunar soil require more the physical characteristics to be matched. Because it is impossible to make a perfect replica of lunar material using terrestrial sources, there will still be some risk introduced into ground testing of hardware and will have to be accounted for in design margins to some extent.

A series of workshops have been held with the lunar simulant users to help identify and refine their needs. The most recent simulant workshop was held in Huntsville, Alabama, in October 2007, and the next workshop is planned for the first quarter of calendar year 2009. These workshops have assisted in communications among the simulant users, simulant developers, and program and project management. They have also provided a conduit for defining user needs, including properties of importance and interest and accuracies or fidelities needed. Timelines, forecasting quantities and schedules are also established. By keeping the source for lunar simulant material centralized, NASA can help defray the cost of development, as well as enable the equitable comparison of different technologies. In cases where multiple types of simulants are available for use, situations will arise where test results will vary even using the same or very similar types of technologies and procedures. Thus, making it very difficult to compare results and decide what technology or hardware should be selected from a pool of several choices. To help alleviate this problem and to "level the playing field" MSFC has also developed a tool that utilizes defined Figures of Merit (FoM). The FoMs allow a user to compare the properties of two or more simulants to each other, or a simulant against actual lunar sample data. The tool will provide a score between zero and one and allow the user to determine the appropriateness of the simulant for particular applications or to understand after use of the simulant how close the simulant properties are to actual regolith. Currently, there are four Figures of Merit; these are size, shape, composition, and density.

In conjunction with being able to compare two or more simulants with each other or an Apollo sample, developing processes to be able to manufacture repeatable simulants are important. These processes will allow simulants to be produced in a timely and cost effective manner with little variability between and among batches. The United States Geological Survey (USGS) is currently working with NASA in the development of these processes. Feedstock development; crushing, grinding, blending, and mixing of the material; packaging, shipping and handling; characterization and testing are all important areas that must be understood and addressed before simulants can be manufactured in a reproducible manner. The constituents of lunar material are replicated in the simulant recipe. Recipes can be modified based on user requirements to produce a "designer" simulant to meet their particular needs.

\section{Oxygen and Other Volatiles}

On the lunar surface, some amount of regolith will need to be excavated and, in most cases, transported to the site where resources will be extracted. Some concepts under development involve the use of mobile equipment, moved to the mining site, which performs processing at that location. Other NASA centers involved in ISRU 
activities are addressing these mining and transportation functions. Depending on the method of oxygen extraction to be used, pre-processing will occur on the material which involves milling, material separation, or other processes. These separation processes, known as beneficiation, will change the regolith into a physical form that is easier to process and/or increase the concentration of the desired elemental fractions. The material not desired will be removed and can be discarded as waste, but will be more likely used for other purposes such as the construction of bricks, berms, or other physical structures or used as radiation shielding material.

The beneficiated material will be processed to remove oxygen and possibly other volatile chemicals that are bound within the regolith components. The oxygen produced from local lunar materials will be used for life support and/or propulsion for applications such as for fuel for the ascent vehicle from the lunar surface. It is also anticipated that water will be produced from lunar resources. The oxygen extracted from the regolith will be combined with hydrogen either scavenged from propellant tanks brought up on the launch vehicle or supplied as up-mass to make water. The use of lunar regolith to produce water and oxygen provides a tremendous "mass leverage" for lunar missions. Depending on the landing location and the soil samples chosen, portions of the lunar regolith are as much as $40 \%$ oxygen by mass. The regolith composition or mineralogy will determine how the oxygen is bound in the material. The composition of regolith varies all over the moon and especially between sites located along the equator and sites near the poles. While it will not be the most efficient use of resources to capture all of the oxygen contained within each regolith sample collected, a substantial fraction can be captured and utilized.

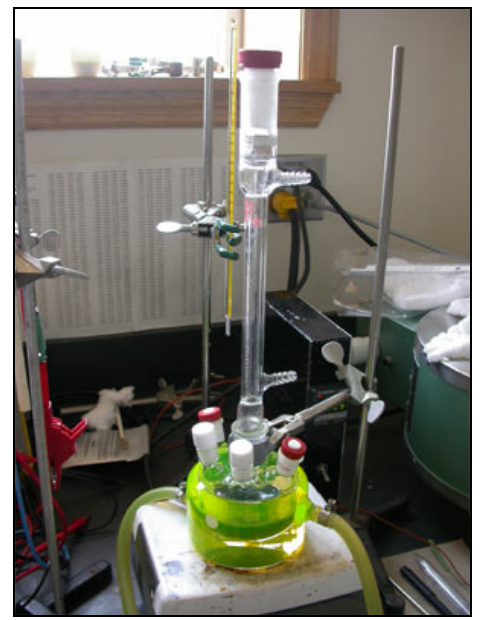

Figure 6. Ionic Liquids Lab Set-Up

Several different methods of extracting oxygen from the lunar material are being studied and researched in MSFC's and other's labs now. As the technologies mature and further knowledge is gained about lunar surface and sub-surface conditions which drive excavation decisions, the oxygen extraction choices will continue to be narrowed down. Eventually, one or more technologies will be chosen for field tests on the lunar surface, and a method will be selected for production of oxygen during lunar missions. The competing technologies have differing resource requirements such as power, mass, processing time, amount of regolith required, etcetera and will be part of the criteria used to down-select methods for further development. Technologies for extraction of oxygen being researched at MSFC include electrolysis utilizing an acidic ionic liquid (see Figure 6); extraction of water from permafrost utilizing microwaves (assuming water ice is bound in the regolith at the poles) (Figure 7); and molten oxide electrolysis (MOE) ${ }^{5}$ with partner Kennedy Space Center. Other technologies are being studied at other NASA Centers, industry, and academia.

Molten Oxide Electrolysis or MOE involves heating beneficiated regolith to a relatively high temperature (current research specifies approximately $1600^{\circ} \mathrm{C}$ ). An electrical current is then run through the pool of molten materials and electrolysis is performed, with oxygen being produced at the anode and metals at the cathode. Because the method involves relatively high temperatures and, thus, a strong power/thermal source, recycling of the heat is a priority. This research, led by the Kennedy Space Center, is being conducted with the Massachusetts Institute of Technology, the Ohio State University, and MSFC. The
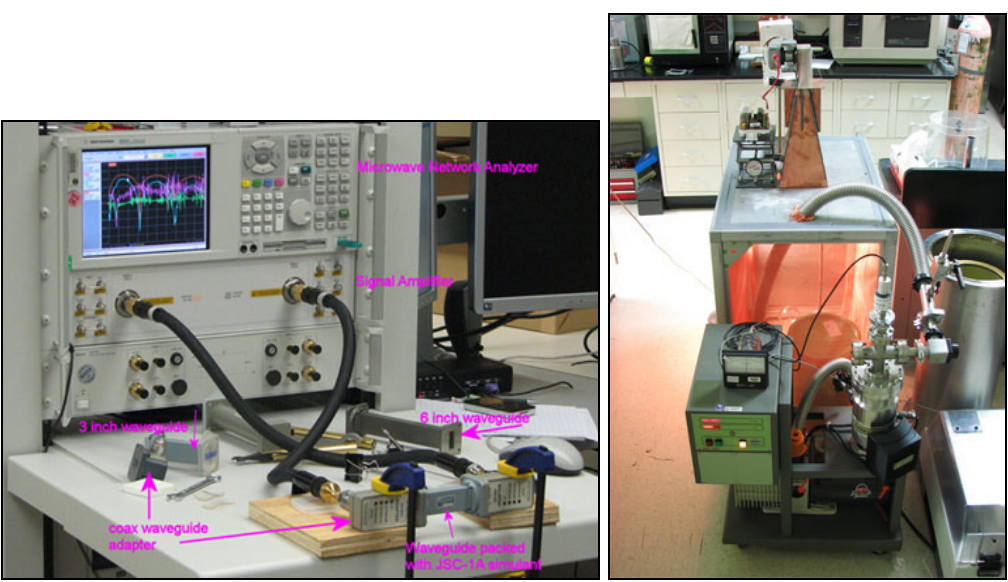

Figure 7. Water Extraction Microwave Lab Set-up Marshall Space Flight Center is currently leading the portion of the system that handles the molten materials and other materials. Examples of material compatibility study samples are shown in Figure 8 below. 


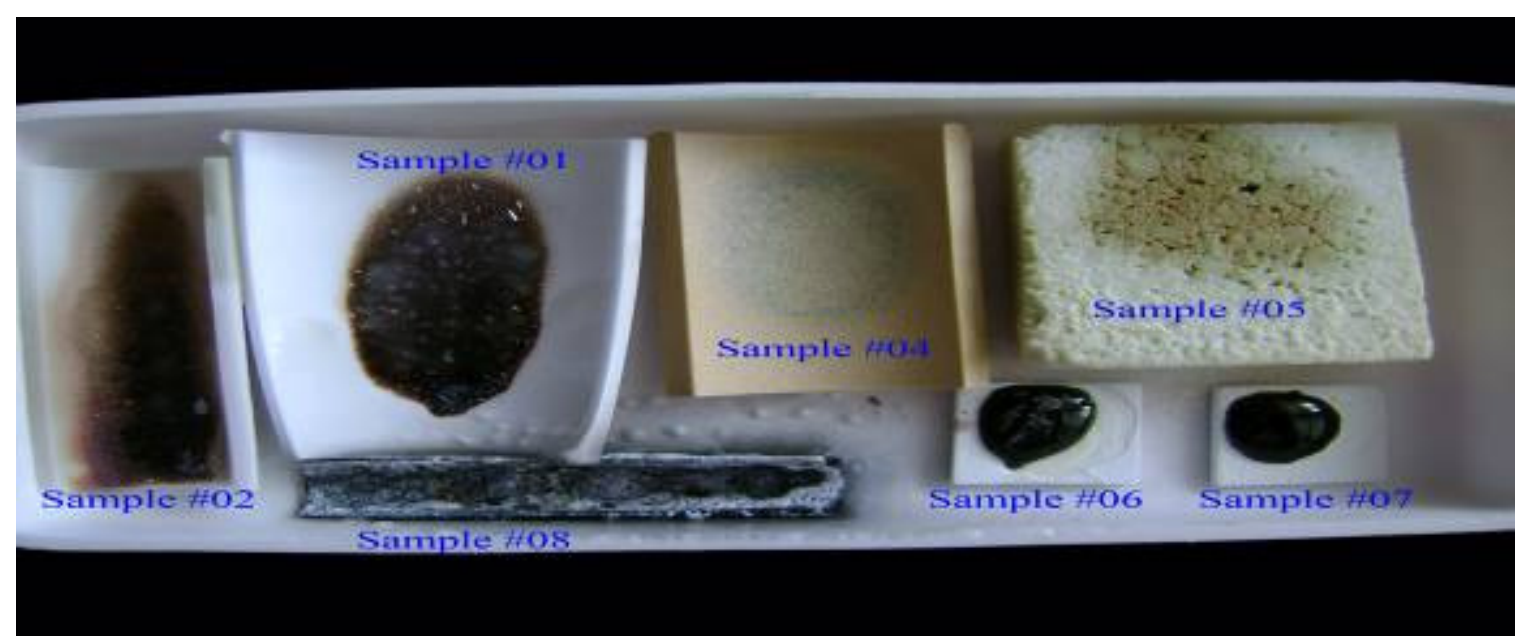

Figure 8. Molten Oxide Electrolysis (MOE) Material Compatibility Test Samples

Another approach to oxygen extraction from lunar material involves dissolving the regolith in an acidic ionic liquid. Because ionic liquids have essentially no vapor pressure, use of this class of materials has advantages for use in the vacuum environment of space. Water is produced from the oxygen bound in the regolith and the hydrogen from the acid. The water is distilled and can be condensed into another ionic liquid electrolyte or electrolyzed within the same ionic liquid. In either case, the water is electrolyzed, and oxygen is produced at the anode. The hydrogen that is produced at the cathode is used to regenerate the ionic liquid.

Data from the Clementine probe in 1994 and the Lunar Prospector probe in 1999 gave indication of a permafrost layer, containing water ice, trapped below the lunar surface in the permanently shadowed craters at the poles. Controversy continues over the presence of such ice, but the Lunar Crater Observation and Sensing Satellite (LCROSS) mission which is scheduled for launch in late 2008, is expected to help resolve these questions. Assuming the permafrost does exist, experiments have been performed at MSFC with simulants to show that microwaves will penetrate a meter or more into the lunar regolith and will heat the regolith (simulant) adequately to create enough vapor pressure to enable the water vapor to rise to the surface. Thus, this method could be used to extract water from the permafrost layer without digging or disturbing the soil via excavation. Once the water vapor is collected, it can be electrolyzed to produce both oxygen and hydrogen. This method, of course, will require work in the extreme cold of the shadowed carters, and does not produce any metallic byproducts for use as raw materials for fabrication.

For each of these technologies, experiments have been conducted to demonstrate feasibility, but each requires further technology development work in order to raise the Technology Readiness Level (TRL). Each method needs to be scaled up to show feasibility for mass production, and requires further testing under conditions matching the lunar surface as closely as possible. This technology development work will require the regolith simulants being developed by MSFC.

\section{Metals Extraction}

As noted, some methods of oxygen extraction being studied at MSFC, as well as those being researched elsewhere, produce considerable quantities of byproducts that are (relatively) enriched in metals such as iron, titanium, and aluminum ${ }^{6}$. These metals were found in abundance in some Apollo samples and can be used as resources for "growing" a lunar base or outpost. In the early stages of a lunar base, it may not be possible or at least it may be too risky to rely solely on this material for metal feedstock to be used in fabrication; thus, it may be necessary to provision feedstock or binder from Earth. However, as the lunar base matures, more equipment is added, and risks are proved to be minimal, the advantage of mining and utilizing these metals in the regolith becomes even more attractive as the up-mass required to deliver spares and other items is saved and can be used to bring scientific experiments and other necessary supplies. 
Some post-processing of the metals may be necessary to meet the quantity, composition or purity, and size/shape as needed for fabrication. Current technology development has focused on the early days of the lunar outpost, when it is assumed that feedstock will be provisioned. But it will be necessary to develop the technologies to prepare this material for further use, and these technological developments are part of the overall technology development "roadmap".

\section{Utilization of Metals}

The ability to produce spare and replacement parts or tools or other needed items at the lunar outpost can reduce risk of loss of mission (or even life) by providing a capability for quicker replacement and "return to operations". The ability to produce unique parts or tools on site as unforeseen contingencies arise will provide for additional flexibility of missions. Also, reducing the need for provisioned spares will increase available mass for depositing other payloads thereby enhancing mission capabilities.

MSFC has been developing the Electron Beam Melting (EBM) process for production of parts using metallic powders. This process involves melting a layer of the powder and forming a two-dimensional "slice" of the part, then adding another layer, then another, eventually producing a three-dimensional part from a series of twodimensional layers. The parts produced have full density and near-net shape, avoiding the pits and voids found in other powder-based additive manufacturing techniques. Current mechanical properties testing have revealed good results. The surface finish, however, still requires some improvement.

MSFC has produced titanium parts utilizing this process (shown in Figure 9) and performed mechanical testing on them. The parts have shown very good physical properties, including strength, fracture and fatigue, very close to that of cast parts. Development work is also proceeding for production of parts from aluminum alloy materials.

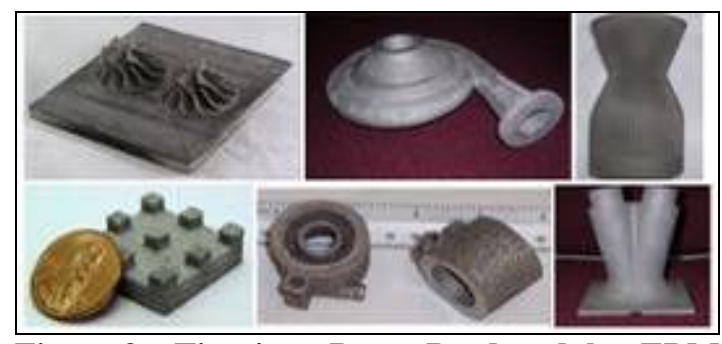

Figure 9. Titanium Parts Produced by EBM Process

To build parts using EBM, a Computer-Aided Design (CAD) file is processed by "slicing" it into layers, each layer defining a two-dimensional path. The electron beam gun follows this path, then the powder bed is lowered by the thickness of one layer and a rake system spreads new powder on the bed. Each layer is successively melted and attaches to the previous layer until the entire part is built.

This technology offers extensive flexibility in that designers have more freedom to create complex geometries, including such things as internal cavities, which could not be created in a single piece by traditional manufacturing methods. Thus, assemblies can be created with fewer parts because they can be combined in the design phase. This process also provides for faster turn-around of parts production which is an added advantage for items needed quickly.

Because the process utilizes an electron beam for the melting, EBM must operate in a vacuum. Of course, vacuum is one resource that is in plentiful supply on the lunar surface. The ability to quickly and easily evacuate the build chamber is another advantage of the EBM process for lunar operations.

Initially, the metal powder that is used by the EBM process will most likely need to be provisioned from Earth until the infrastructure is such that a sufficient quantity of the correct purity and physical characteristics can be produced locally. Even so, the mass required for operations is substantially less than necessary to provision all the needed spare parts individually. The mass advantage only increases as the powders can be produced and refined on the moon.

The technologies for additive manufacturing are more complex than for subtractive manufacturing (i.e., milling and lathing a part out of bar or other solid stock material), but the mass savings of material or lack of waste is noticeable. The mass of feedstock (powder) consumed is only slightly above that of the finished part when EBM is used, but with subtractive methods the feedstock mass (and waste) is much greater. However, technology trade studies $^{7}$ performed at MSFC showed that no one fabrication can "do it all". It is anticipated that the optimum 
approach for fabrication on the lunar surface may involve a hybrid system with a combination of additive and subtractive technologies to arrive at the most effective capability.

Although EBM is a "proven" technology and the hardware is sold commercially, work is required to make the processing of materials certified and ready for lunar use. Initially, parts will be built on earth, for use on Earth, and certified for use in current projects and applications. Work is currently being performed by MSFC and its partners, The Boeing Company and North Carolina State University (NCSU), to produce that path to certification. Later, this same method will be used to certify the process for operating on the lunar surface and allow it to be applied to parts produced in situ on the moon.

The ultimate goal would be the ability to utilize lunar regolith with little or no pre-processing, or the byproducts of an oxygen extraction process with no additional processing, as the input material to a fabrication process. While this vision may never be fully realized due to the chemical and/or physical characteristics of the material, it may be possible to produce parts with a small percentage of binder material (such as provisioned aluminum powder) to allow the regolith to properly melt in order to produce a part of adequate strength. As a simple test run, some NULHT-1M lunar simulant was used recently as feedstock. Contrary to expectations, a light sintering did occur with the EBM process. Further tests were performed with a small fraction of aluminum binder material, and multi-layer builds has been achieved. Additional work in the way of trying different machine parameter settings and various simulant/binder ratios is planned to advance this capability and assess viability for future use on the lunar surface.

\section{Inspection of Parts}

In most cases, parts built in situ will need to be inspected on site before being released for use. Some forms of nondestructive evaluation (NDE) will need to be performed to determine the integrity of the parts for their intended use. On Earth, many types of NDE methods are employed for parts inspections. Method(s) are chosen for the specific application, taking into account factors such as the nature of the part; the material from which it was made; geometry, size and type of defect to be detected; and the environment. For lunar applications, it is necessary to minimize the suite of technologies to be employed due to limitations on equipment to be launched and available crew time.

The MSFC team performed several studies to select NDE technologies that fit several criteria. These criteria included: the potential for development into portable or handheld devices; applicability to a wide range of materials; suitability for operations in the lunar environment; and "ease-of-use" with less dependence on crew interaction. Technologies under develop for lunar applications at MSFC include a Microwave-Millimeter Wave Camera System and portable Laser Ultrasonics System (see Figures 10 and 11, respectively).

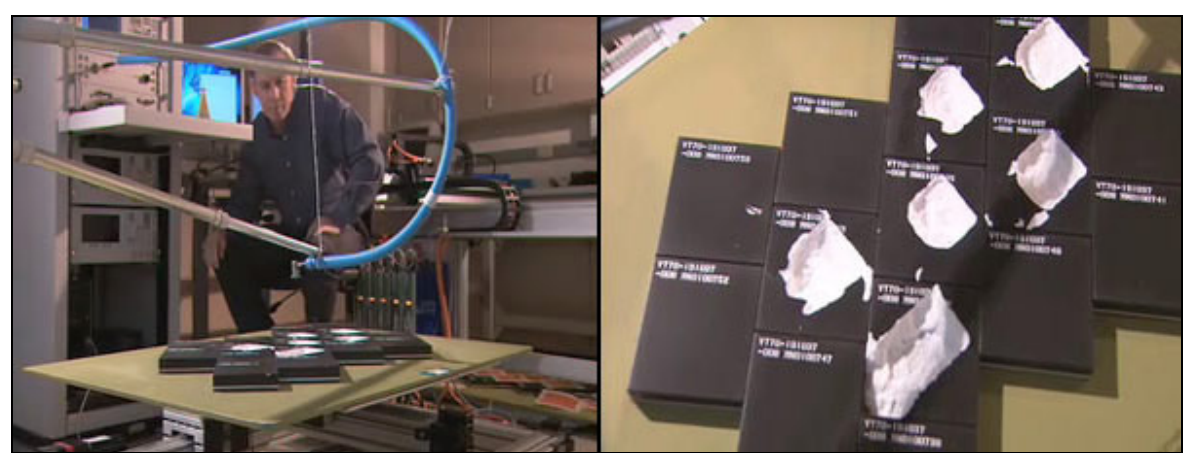

Figure 10. Microwave/Millimeter Wave System Lab Set-up
Microwave scanning systems are used for a range of materials, but are especially used for applications such as discriminating detail inside non-metallic, di-electric materials like thermal protection systems foam on the Space Shuttle, for example. However, scanning systems are large and scans often require hours to complete. The systems under

development would utilize the same technology, but now the devices are being designed to be handheld and allow for real-time imaging thereby saving time. 

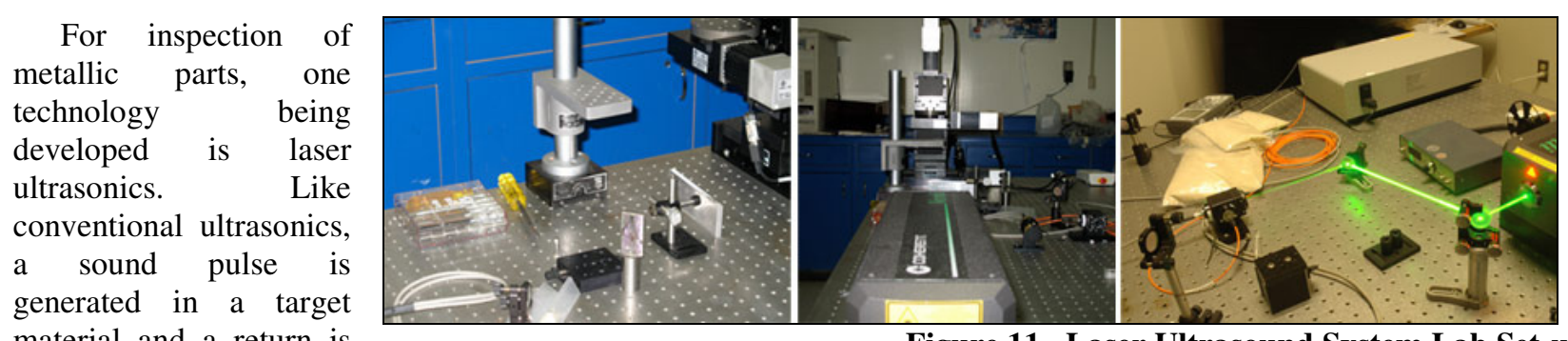

material and a return is measured and analyzed

Figure 11. Laser Ultrasound System Lab Set-up to determine information about the interior and surface of the target object. Unlike conventional ultrasonics, laser ultrasonics can be performed in a non-contact manner and works equally well in a vacuum These two discriminating factors led to the selection of the laser ultrasound as a candidate for lunar technology development. Current development work at MSFC involves testing it on aluminum parts that have been produced by the EBM process.

\section{Conclusions}

To provide for a long-term human presence on the moon utilizing the resources found there will be of utmost importance. One of the primary reasons is the prohibitive expense of transporting mass to the lunar surface. To make use of these lunar resources, they must be first understood and studied in order to take full advantage of them and address any and all challenges also presented by utilizing the in situ resources. Process and technology developments are underway now, and these require the development of high quality simulants to use on Earth to reduce risk to humans and hardware. The MSFC ISRU team is developing various lunar simulants to meet the different users' needs that support all areas of development for the lunar missions.

The single most important development that is required for enhancing, and in some cases enabling, sustained human presence on the lunar surface is having the capability to extract oxygen, water (if present in water ice at the poles), and metals from the lunar regolith. The NASA/Marshall Space Flight Center ${ }^{8}$ is developing or assisting other centers in developing several extraction technologies, as well as the EBM process that will utilize the metals extracted to fabricate items. To ensure the parts produced are of the right quality and safe to put into operations, they will need to be inspected for defects. Non-destructive evaluation technologies are also being developed by MSFC to enable this also.

Each of the technologies being developed requires further efforts to increase the technology readiness level in order to meet the Constellation Program's schedule. The potential for extending the capabilities of the lunar missions utilizing ISRU technologies is enormous. These technologies will give the future lunar inhabitants the same thing that the Native Americans provided the Pilgrims: the capability to "live off the land" and allow them to not only survive, but to thrive and prosper.

\section{Acknowledgments}

Portions of this work were performed in support of the George C. Marshall Space Flight Center under the Teledyne Brown Engineering Systems Development and Operations Support (SDOS) contract (No. NAS802060).

\section{References}

${ }^{1}$ Stanley, D., Cook, S., and Connolly, J., "NASA's Exploration Systems Architecture Study,” NASA TM-2005214062, November 2005.

${ }^{2}$ Charles Meyer, "The Lunar Sample Compendium”, http://curator.jsc.nasa.gov/lunar/compendium.cfm

${ }^{3}$ Eric M. Jones, “Apollo Lunar Surface Journal”, http://history.nasa.gov/alsj/frame.html

${ }^{4}$ Sanders, G., K. Romig, W. Larson, D. Rapp, K. Sacksteder, D. Linne, P. Curreri, M. Duke, B. Blair, L. Gertsch, D. Boucher, L. Clark, E McCollough, R. Zubrin, "Results from the NASA Capability Roadmap Team for In-Situ Resource Utilization," International Lunar Conference, September 20, 2005, Toronto , Canada. 
${ }^{5}$ Curreri, P.A., Sen, S., and Sadoway, D.R., "Process Demonstration For Lunar In Situ Resource Utilization Molten Oxide Electrolysis,” NASA TM-2006-214600, 2006.

${ }^{6}$ Stefanescu, D.M., R.N. Grugel and P.A. Curreri, "In situ Resource Utilization for Processing of Metal Alloys on Lunar and Mars Bases," in Space 98 The Sixth International Conference and Exposition on Engineering, Construction and Operations in Space, Rodney G. Galloway and Stanley Lokaj, editors, American Society of Civil Engineers, Reston, VA, pp. 266 - 274, 1998.Van Vuuren, D.S., A.D. Engelbrecht and T. D. Hadley, JOM 57 (2005).

${ }^{7}$ Howard, R., Patterson, B., Gilley, S., and Kennedy, J., "Fabrication Technology Options Trade Study," Teledyne Brown Engineering, 480RPT0190, 2004.

${ }^{8}$ Marshall Space Flight Center, In Situ Resource Utilization website, http://isru.msfc.nasa.gov 